\title{
Use of speech-modulated noise adds strong "bottom-up" cues for phonemic restoration
}

\author{
JAMES A. BASHFORD, JR., RICHARD M. WARREN, and CHRISTOPHER A. BROWN \\ University of Wisconsin, Milwaukee, Wisconsin
}

\begin{abstract}
When deleted segments of speech are replaced by extraneous sounds rather than silence, the missing speech fragments may be perceptually restored and intelligibility improved. This phonemic restoration $(\mathrm{PhR})$ effect has been used to measure various aspects of speech processing, with deleted portions of speech typically being replaced by stochastic noise. However, several recent studies of $\mathrm{PhR}$ have used speech-modulated noise, which may provide amplitude-envelope cues concerning the replaced speech. The present study compared the effects upon intelligibility of replacing regularly spaced portions of speech with stochastic (white) noise versus speech-modulated noise. In Experiment 1, filling periodic gaps in sentences with noise modulated by the amplitude envelope of the deleted speech fragments produced twice the intelligibility increase obtained with interpolated stochastic noise. Moreover, when lists of isolated monosyllables were interrupted in Experiment 2, interpolation of speech-modulated noise increased intelligibility whereas stochastic noise reduced intelligibility. The augmentation of PhR produced by modulated noise appeared without practice, suggesting that speech processing normally involves not only a narrowband analysis of spectral information but also a wideband integration of amplitude levels across critical bands. This is of considerable theoretical interest, but it also suggests that since PhRs produced by speech-modulated noise utilize potent bottom-up cues provided by the noise, they differ from the PhRs produced by extraneous sounds, such as coughs and stochastic noise.
\end{abstract}

In everyday listening, speech signals of interest are frequently interrupted by extraneous sounds. Yet, when interruptions are brief, the speech may appear intact and intelligibility may remain high. Laboratory studies have shown that this phenomenon reflects more than the ability to use partially masked speech accompanying the interrupting sound-indeed, when part of a word is completely removed and replaced by an extraneous sound such as a cough or stochastic noise, listeners perceptually restore the missing segment, based on information preceding and following the interruption, and they cannot distinguish between the portions of speech that are physically present and those that have been perceptually synthesized (Samuel, 1981 a; Warren, 1970; Warren \& Obusek, 1971; Warren \& Sherman, 1974).

The perceptual synthesis of absent speech, which is known as phonemic restoration ( $\mathrm{PhR}$ ) (Warren, 1970), represents the linguistic adaptation of a more general auditory process that restores continuity by subtracting a portion of the neural input produced by the interrupting sound and using it to reconstruct the missing signal frag-

This work was supported by a grant to the second author from the National Institutes of Health (DC00208). The experiments reported were outlined in a paper presented at the 127th meeting of the Acoustical Society of America, with a brief abstract appearing in the Journal of the Acoustical Society (1994, Vol. 95, No. 5(2), p. 2975). The authors gratefully thank Makio Kashino and Keri Riener for technical assistance related to this work. Correspondence should be addressed to J. Bashford, Department of Psychology, University of Wisconsin, Milwaukee, Wisconsin 53201 (e-mail: bashford@alphal.uwm.edu). ment (Warren, 1984; Warren, Bashford, Healy, \& Brubaker, 1994). This process of "auditory reallocation" requires a spectral overlap between the interrupter and the interrupted signal, and thus ensures that the substrate for perceptual synthesis will be available only when the interrupting extraneous sound is capable of masking the signal (Warren et al., 1994). When this basic acoustic requirement is met, and when there is sufficient signal context to guide synthesis, reallocation can produce apparent continuity of both speech and a variety of nonverbal sounds, including steady-state signals such as pure tones (Houtgast, 1972; Miller \& Licklider, 1950; Thurlow, 1957; Warren, Obusek, \& Ackroff, 1972) and dynamic time-varying signals such as frequency glides (Ciocca \& Bregman, 1987; Dannenbring, 1976; Kluender \& Jenison, 1992) and music (DeWitt \& Samuel, 1990; Sasaki, 1980; Verschuure, 1978).

The present study deals with the verbal form of auditory reallocation, phonemic restoration, and the contribution to phonemic restoration made by different types of information: information preceding and following the interruption and information available during the interruption. Previous investigations have focused on restorations occurring when a portion of speech is completely replaced by noise, and those studies have shown that, under a variety of conditions, information provided by the intact portions of speech is sufficient to restore the appearance of continuity (Bashford, Meyers, Brubaker, \& Warren, 1988; Bashford \& Warren, 1979, 1987b; Layton, 1975; Miller \& Licklider, 1950; Samuel, 1981a, 1981b, 1987, 1991; Samuel \& Ressler, 1986; Verschuure, 1978; Warren, 1970; Warren \& Obusek, 1971; Warren \& Sherman, 1974) and to restore 
intelligibility (Bashford, Riener, \& Warren, 1992; Bashford \& Warren, 1979, 1987a; Cherry \& Wiley, 1967; Holloway, 1970; Powers \& Wilcox, 1977; Verschuure, 1978; Verschuure \& Brocaar, 1983). However, interruption under normal listening conditions involves the mixing of an extraneous sound with the speech signal, and thus, under conditions of incomplete masking, allows for possible contributions to restoration made by remnants of the speech signal that are available during the interruption. The present study investigated this topic by contrasting restoration effects produced by interpolated stochastic noise, which contributes no information concerning the identity of the missing fragments, and interpolated speechmodulated noise. While speech-modulated noise provides no formant information, it does duplicate in detail the broadband amplitude envelope of the replaced speech fragments.

There were several reasons for choosing speechmodulated noise as the interrupting stimulus in this study. First, it has been hypothesized that the broadband amplitude envelope of speech might serve as a rich source of suprasegmental and phonemic information (e.g., Erber, 1979), although relatively little is currently known about the role of this information in normal speech perception (for recent reviews, see Repp, Frost, \& Zsiga, 1992, and Rosen, 1992). Previous studies have shown that speechmodulated tones can provide cues concerning articulation rate (Gordon, 1988) and syllable numerosity (Remez \& Rubin, 1990), and both modulated tones and modulated noises have been found to provide supplementary information for lipreading (Blamey, Martin, \& Clark, 1985; Breeuwer \& Plomp, 1984, 1986; Erber, 1972; Grant, Ardell, Kuhl, \& Sparks, 1985; Grant, Braida, \& Renn, 1991, 1994; Lemay \& Braida, 1994). In addition, it has been shown that listeners can recognize the correspondence between the amplitude envelope of a word that has been superimposed upon white noise and either the printed version of that word (Frost, 1991; Frost, Repp, \& Katz, 1988) or the articulatory gestures associated with its production (Repp et al., 1992). Other studies that have presented modulated noise as the lone source of verbal information have shown that listeners can learn to recognize the amplitude envelopes of syllables (van Tasell, Soli, Kirby, \& Widin, 1987) and words (Horii, House, \& Hughes, 1971) when stimuli are drawn from small, closed sets of items. However, when stimuli are drawn from a large unknown set, the stand-alone intelligibility of speech-modulated noise is essentially zero (Frost et al., 1988). ${ }^{1}$

It is quite important, from a methodological standpoint, to know whether speech-envelope information conveyed by modulated noise can be combined with information from intact speech to augment phonemic restoration. Speechmodulated noise has been recommended (Horii et al., 1971; Schroeder, 1968) and used (e.g., Beck \& Speaks, 1993; Festen \& Plomp, 1990) as an alternative to stochastic noise for speech masking because it permits the experimenter to select any desired signal-to-noise $(\mathrm{S} / \mathrm{N})$ ratio and maintain this value moment-to-moment despite fluctuations in speech amplitude. However, if the modulated noise also provides useful information concerning the speech signal, its role as a masking stimulus is seriously compromised. Furthermore, and of more direct relevance to the present study, speech-modulated noise has been used previously as a replacement stimulus in studies of phonemic restoration (Repp, 1992; Samuel, 1987, 1991; Samuel \& Ressler, 1986; Trout \& Poser, 1990) and in studies employing the gating paradigm (Salasoo \& Pisoni, 1985; Walley, 1988).

The present experiments contrasted the effects of stochastic and speech-modulated noise upon the restoration of intelligibility. Speech signals were subject to periodic interruptions, and restoration was measured in terms of the increase in keyword repetition accuracy that resulted when regularly spaced gaps in the speech were filled with either stochastic or speech-modulated noise. Both types of interpolated noise had flat (white) long-term spectra and bandwidths that matched that of the speech signals. In Experiment 1 , separate groups of listeners were presented with sentences that were interrupted either by silence or by one of the two types of noise presented at one of three S/N ratios: $0,-8$, or $-15 \mathrm{~dB}$. The $0-\mathrm{dB} \mathrm{S} / \mathrm{N}$ ratio matched the noise level employed in earlier studies that have used speech-modulated noise as a replacement stimulus in gating or phonemic restoration paradigms (e.g., Salasoo \& Pisoni, 1985; Samuel \& Ressler, 1986). The -8- and - 15$\mathrm{dB} S / \mathrm{N}$ ratios corresponded to typical levels used for interpolated stochastic noise in earlier studies of multiple restorations occurring with periodically interrupted speech (e.g., Bashford et al., 1992; Verschuure \& Brocaar, 1983). To foreshadow: Experiment 1 showed that the restoration effects obtained when sentences were interrupted by speech-modulated noise far exceeded the effects obtained with stochastic noise. Therefore, it was of interest to extend the study to isolated monosyllabic words, which had previously been found not to increase in intelligibility with the interpolation of stochastic noise (Bashford \& Warren, 1987a; Bashford et al., 1992; Dirks \& Bower, 1970; Miller \& Licklider, 1950). In Experiment 2, it was found that the addition of stochastic noise to gaps in words decreased intelligibility, whereas the addition of speech-modulated noise increased intelligibility.

\section{GENERAL METHOD}

The sentences employed in Experiment 1 and the isolated words employed in Experiment 2 were produced by a male speaker and bandpass filtered from $100 \mathrm{~Hz}$ to $8 \mathrm{kHz}$ (Rockland Model 852 filter producing slopes of $48 \mathrm{~dB} /$ octave) before initial recording on audiotape using an Ampex 440-C eight-track recorder operating at 15 ips (see Bashford \& Warren, 1987a, for further details). For use in the present experiment, playback of the speech signals was digitized (22-kHz sampling frequency, 16-bit quantization), and each of the sentences and isolated monosyllables was individually rescaled in level so that, upon playback, the peak amplitudes of all items varied by less than $0.2 \mathrm{~dB}$ SPL, as measured by a Brüel \& Kjar Model 2230 precision integrating sound-level meter. The amplitude-adjusted speech files were then used to create stereo files that had one track consisting of the speech stimuli (i.e., sentences or words, separated by 3 -sec silent pauses) and a second track consisting of bursts of ei- 
ther speech-modulated or stochastic noise occurring simultaneously with the speech. The stochastic noise files were created by copying the speech files, mapping the boundaries of individual sentences or words, using a waveform editor, and then replacing the verbal stimuli with segments of white noise (Brüel and Kjær Model 1405 noise generator). The speech-modulated noise tracks were produced by copying the speech files and randomly reversing the polarity of individual sample points with a probability of 0.5 (Schroeder, 1968). (It should be noted that, in our experiments, the procedures used to produce the noise files resulted in a time alignment of the onsets and offsets of the speech stimuli and corresponding noise bursts, thus ensuring that interpolated noise in both the stochastic and speechmodulated noise conditions always replaced speech and did not occur during the interstimulus intervals.) The nature of the speechmodulated noise is illustrated in Figure 1, which provides oscillograms, root-mean-square (rms) amplitude-envelope plots, and spectrograms for both the digitized Central Institute for the Deaf (CID) sentence, "Come here when I call you," and the speech-modulated noise derived from that sentence. As shown in the spectrograms, formant structure is destroyed by the randomization of polarity. However, the waveforms are quite similar, and the rms envelopes are, of course, equivalent.

For production of the final stimuli, the stereo digital files were played back and recorded on audiotape (Otari Model MTR-10 twotrack recorder operating at $15 \mathrm{ips}$ ) with the noise tracks subjected to an intervening bandpass filtering identical to that of the speech signals ( $100 \mathrm{~Hz}$ to $8 \mathrm{kHz}$ with $48-\mathrm{dB}$ /octave slopes). During the experiments, the analog playback of the speech and noise channels was passed to the listener's headphones by separate, alternately triggered electronic switches (Coulbourn Model S84-04), which shaped the complementary onsets and offsets of the speech and noise with 10 -msec raised-cosine ramps. The electronic switches ran continuously throughout an experimental session, so that the placement of periodic gaps in the speech signals was completely independent of the speech and determined randomly for each listener in all conditions. For the sentences presented in Experiment 1, the speech signal was on for $150 \mathrm{msec}$ and off for $250 \mathrm{msec}$ of each 400 -msec switching cycle (i.e., periodic interruption rate of $2.5 \mathrm{~Hz}$ ). For the isolated monosyllabic words presented in Experiment 2, the total switching cycle was also $400 \mathrm{msec}$ in duration, but on- and offtimes were matched at $200 \mathrm{msec}$ ( $50 \%$ duty cycle) ${ }^{2}$

Listeners were tested individually in an audiometric room with stimuli delivered diotically through Sennheiser HD 250-Linear headphones. The speech signals were always presented at a peak level of $70 \mathrm{dBA}$, and the interrupting noise (when present) was delivered at a peak level of 70,78 , or $85 \mathrm{dBA}$ SPL.

\section{EXPERIMENT 1}

\section{Method}

Participants. The 140 subjects ( 7 groups of 20 listeners) were native English speakers with no known hearing problems. They were recruited from introductory psychology courses at the University of Wisconsin-Milwaukee and were given money or course credit for their participation in the study.

Stimuli. The speech stimuli were the $100 \mathrm{CID}$ sentences used in several previous studies dealing with the intelligibility of interrupted speech (Bashford et al., 1992; Bashford \& Warren, 1979, 1987a; Powers \& Wilcox, 1977). These sentences are arranged in 10 lists of 10 sentences and were designed to represent "everyday American speech" (Silverman \& Hirsh, 1955), with each list containing 50 phonetically balanced keywords varying in both syllabic composition and positioning within sentences (e.g., "Come here when I call you!" and "Did you forget to shut off the water?"). The average duration of words in the sentences, calculated from sentence durations, was $294 \mathrm{msec}$.
Procedure. Separate groups of 20 listeners were assigned randomly to each of the seven interruption conditions. For one group of listeners, the sentences were interrupted by silent gaps. For the remaining six groups of listeners, the gaps in sentences were filled with either stochastic or speech-modulated noise presented at a level of 70,78 , or $85 \mathrm{dBA}$ SPL. Since the sentences were always presented at a peak level of $70 \mathrm{dBA}$, the resulting peak $\mathrm{S} / \mathrm{N}$ levels were $0,-8$, and $-15 \mathrm{~dB}$ for both types of noise. The order of presentation for sentence sets was blocked and pseudorandomized within conditions so that each list of sentences occurred twice in each serial position across listeners. Individual testing sessions lasted about $20 \mathrm{~min}$, and each listener was presented with only one interruption condition. Listeners were instructed to repeat back each sentence as best they could and were encouraged to guess if they were unsure. The experimenter scored the number of keywords correctly repeated for each sentence.

\section{Results and Discussion}

Figure 2 presents the mean percent intelligibility scores obtained for the seven experimental conditions, averaged across the 10 blocks of CID sentences presented in Experiment 1 . It can be seen that interpolation of stochastic noise at all $\mathrm{S} / \mathrm{N}$ ratios produced a substantial increase in intelligibility relative to the silent-gap baseline condition, and that the interpolation of speech-modulated noise produced an increase approximately twice that of the stochastic noise. Overall, the highest intelligibility was obtained with the speech-modulated noise in the $-8-\mathrm{dB} \mathrm{S} / \mathrm{N}$ condition, which yielded accuracy scores $38 \%$ higher than those in the silent-gap condition.

These observations are supported by statistical analyses. Listeners' accuracy scores for the six noise conditions were subjected to a three-way analysis of variance, which revealed significant main effects of noise type $[F(1,114)=$ $126.9, p<.0001], \mathrm{S} / \mathrm{N}$ ratio $[F(2,114)=8.8, p<.0005]$, and block $[F(9,1026)=35.8, p<.0001]$. All interactions were nonsignificant $(F \leq 2.0, p>.14)$. Subsequent Tukey tests for the effect of $\mathrm{S} / \mathrm{N}$ ratio indicated that the greatest enhancement of intelligibility by both types of noise $(p<$ .01 ) occurred in the $-8 \mathrm{~dB} \mathrm{~S} / \mathrm{N}$ condition. Analysis of the block effect, using Helmhert single-df contrasts (Hays, 1988 ), indicated that repetition accuracy improved consistently across blocks of sentences $(F \geq 6.1, p<.02$ or better) until reaching asymptote at the seventh block. This pattern of improvement, which resulted in an intelligibility increase of about $23 \%$ across blocks, held for all six noise conditions, as indicated by the nonsignificant interactions.

The data from the six interpolated-noise conditions were also compared with the silent-gap baseline condition in a supplemental two-way analysis of variance (7 interruption conditions $\times 10$ blocks of sentences), which revealed significant main effects of interrupter $[F(6,133)=$ 47.34, $p<.0001]$ and block $[F(9,1197)=40.16, p<$ $.0001]$ and a nonsignificant interrupter $\times$ block interaction $[F(54,1197)=0.76, p>.89]$. Subsequent Tukey tests confirmed that intelligibility was higher $(p<.01)$ in each of the interpolated-noise conditions than in the silent-gap condition.

Averaged across $\mathrm{S} / \mathrm{N}$ ratios, there was a $14 \%$ increase in intelligibility produced by stochastic noise in this experi- 


\section{Speech}
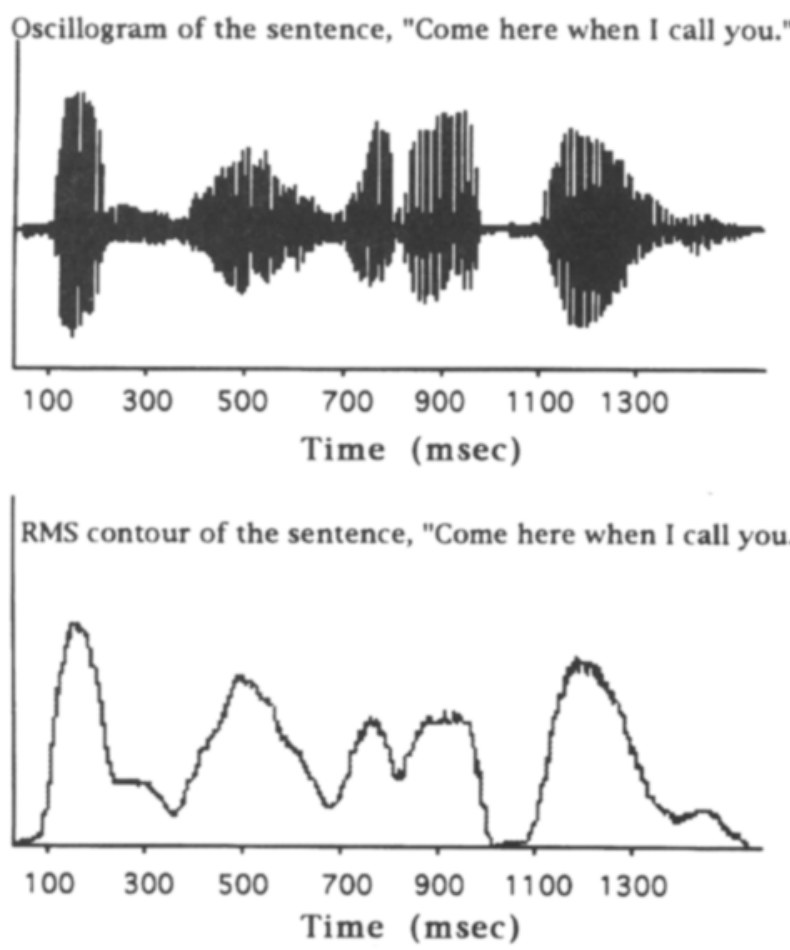

Spectrogram $(0.1$ to $10.0 \mathrm{kHz})$ of the sentence, "Come here when I call you."

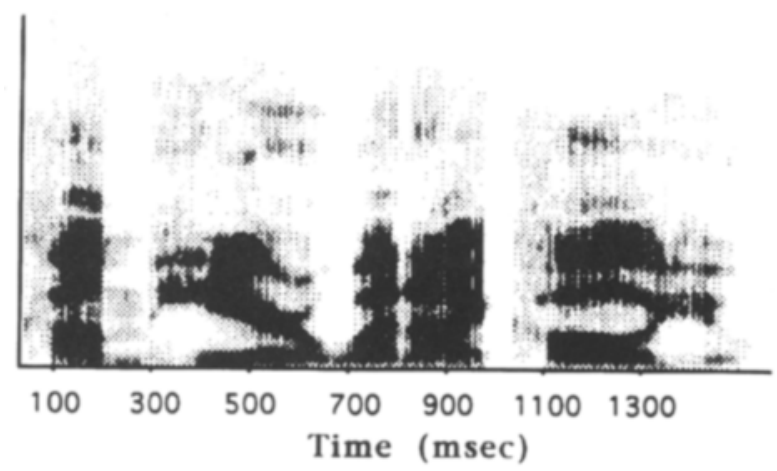

\section{Speech-Modulated Noise}

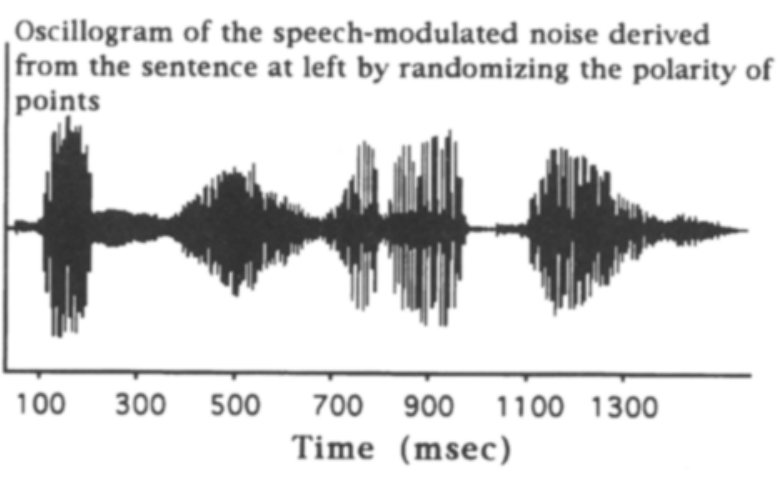
RMS contour of the speech-modulated noise derived from
the sentence at left by randomizing the polarity of Points

Spectrogram $(0.1$ to $10.0 \mathrm{kHz})$ of the speechmodulated noise derived from the sentence at left by randomizing the polarity of points

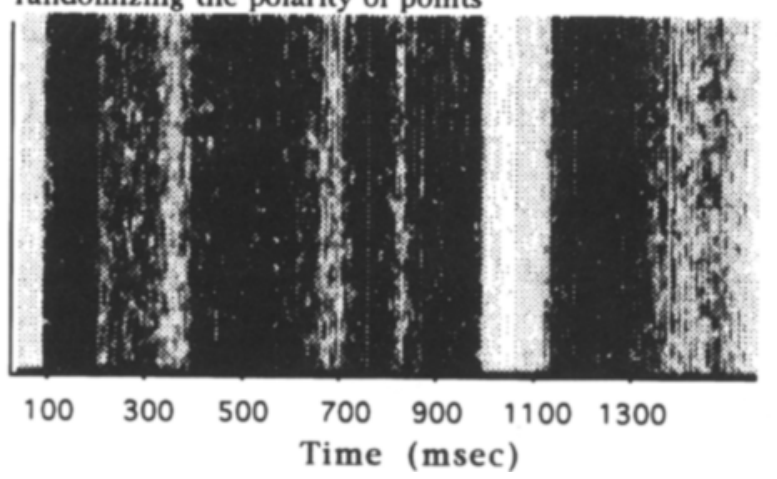

Figure 1. Speech versus speech-modulated noise. Oscillograms, root-mean-square contours, and spectrograms for the sentence "Come here when I call you" and for the speech-modulated noise derived from that sentence by randomly reversing the polarity of points.

ment. This enhancement of intelligibility is nearly identical to that observed in two earlier studies employing similar interruption conditions. Bashford et al. (1992) determined the effect of interpolating $80-\mathrm{dB}$ pink noise $(\mathrm{S} / \mathrm{N}$ ratio $=$ $-10 \mathrm{~dB}$ ) using the same CID sentences and the same 2.5$\mathrm{Hz}$ interruption rate employed in the present study but with a higher $(50 \%)$ duty cycle of speech on-time. Although that duty cycle resulted in higher overall intelligibility scores, the increase produced by the addition of noise was also 14\%. Similarly, Powers and Wilcox (1977) obtained an increase of $14 \%$ for CID sentences inter- rupted at a rate of $2.22 \mathrm{~Hz}$ (225-msec on- and off-times) when gaps were filled with stochastic noise presented at a $0-\mathrm{dB} \mathrm{S} / \mathrm{N}$ ratio. The $0-\mathrm{dB} \mathrm{S} / \mathrm{N}$ condition in the present experiment yielded an intelligibility increase of $13 \%$.

In contrast to the $14 \%$ average increase in sentence intelligibility found in the present study when silent gaps were filled with a potential masker that was unrelated to the speech, there was a $31 \%$ average increase in intelligibility scores when speech-modulated noise filled the gaps. These results indicate that the broadband amplitude envelope of missing sentence fragments, when duplicated by 


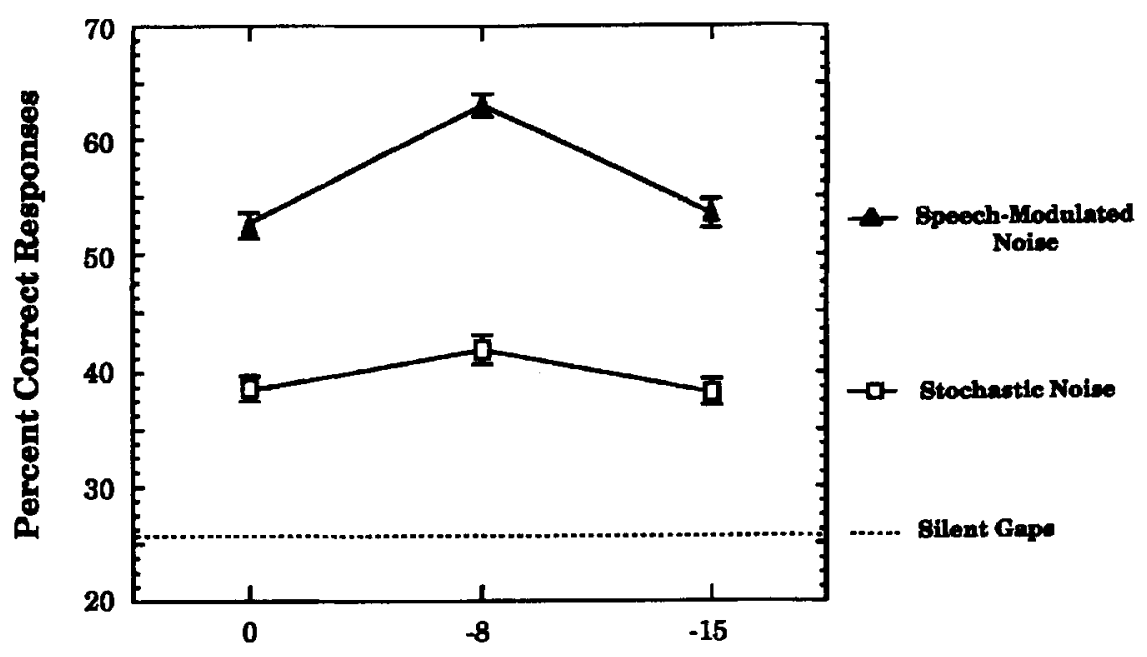

Signal-to-Noise Ratio (dB)

Figure 2. Mean percent repetition accuracy (and standard-error confidence intervals) for keywords in sentences subjected to periodic interruption by silence, stochastic noise, or noise modulated by the amplitude envelope of obliterated speech fragments.

an interrupting noise, provides powerful cues to the identity of the speech segment controlling the noise amplitude. The immediacy of this effect, as indicated by the nonsignificant interrupter $\times$ block interaction, suggests that the use of amplitude-envelope information is a routine component of speech processing, as previously suggested by Erber (1979) and Rosen (1992).

The dramatic augmentation of phonemic restoration occurring when speech-modulated noise rather than stochastic noise was used with sentences suggested that cues provided by speech-modulated noise might provide sufficient bottom-up information to permit restoration of intelligibility for isolated monosyllables. Monośyllabicword lists have previously been found not to provide sufficient context for restoration of intelligibility when interrupted by stochastic noise (Bashford et al., 1992; Bashford \& Warren, 1987a; Dirks \& Bower, 1970; Miller \& Licklider, 1950). This hypothesis was examined in Experiment 2.

\section{EXPERIMENT 2}

\section{Method}

Participants. One hundred and forty additional listeners ( 7 groups of 20 listeners) were recruited from introductory psychology courses at the University of Wisconsin-Milwaukee, using the same qualifications and compensation used for Experiment 1.

Stimuli. The 10 lists of 50 isolated words used in this experiment were phonetically balanced monosyllables (American National Standards Institute, 1971). The interruption rate $(2.5 \mathrm{~Hz})$ and rise/ fall times (10-msec raised-cosine ramps) were as described in the General Method section, but the duty cycle of switching was altered to produce equal on- and off-times of $200 \mathrm{msec}$. The average duration of the monosyllables was $512 \mathrm{msec}$.

Procedure. The procedure was the same as that employed with sentences in Experiment 1 . This included the blocking and pseudo- randomization of presentation order within experimental conditions, which ensured that each set of monosyllables was presented twice in each serial position (block) across listeners and that interruption of the words occurred at randomly determined positions that differed for each listener.

\section{Results and Discussion}

Figure 3 presents the mean percent intelligibility scores obtained for the seven experimental conditions, averaged across the 10 blocks of isolated monosyllables. It can be seen that filling gaps in monosyllables with stochastic noise reduced intelligibility, while the interpolation of speech-modulated noise enhanced intelligibility.

Listeners' accuracy scores for repetition of monosyllables in the six noise conditions were subjected to a threeway analysis of variance, which revealed significant main effects of noise type $[F(1,114)=198.74, p<.0001], \mathrm{S} / \mathrm{N}$ ratio $[F(2,114)=12.19, p<.0001]$, and block $[F(9,1026)=$ $17.20, p<.0001]$. There was also a significant noise type $\times \mathrm{S} / \mathrm{N}$ ratio interaction $[F(2,114)=13.45, p<.0001]$. The remaining interactions were nonsignificant $(F \leq 0.85$, $p>.60)$. A simple-effects analysis of the noise type $\times \mathrm{S} / \mathrm{N}$ ratio interaction indicated that the effect of $\mathrm{S} / \mathrm{N}$ ratio upon intelligibility was significant with interpolated stochastic noise $[F(2,57)=21.96, p<.0001]$ but not with speechmodulated noise $[F(2,57)=1.77, p>.15]$. Subsequent Tukey tests indicated that the S/N-ratio effect for stochastic noise was due to a greater reduction of intelligibility $(p<.01)$ in the $-15-\mathrm{dB}$ condition.

The data from the six interpolated-noise conditions were compared with the silent-gap baseline condition in a twoway analysis of variance ( 7 interruption conditions $\times 10$ blocks of sentences), which revealed significant main effects of interrupter $[F(6,133)=44.41, p<.0001]$ and block $[F(9,1197)=19.58, p<.0001]$ and a nonsignifi- 


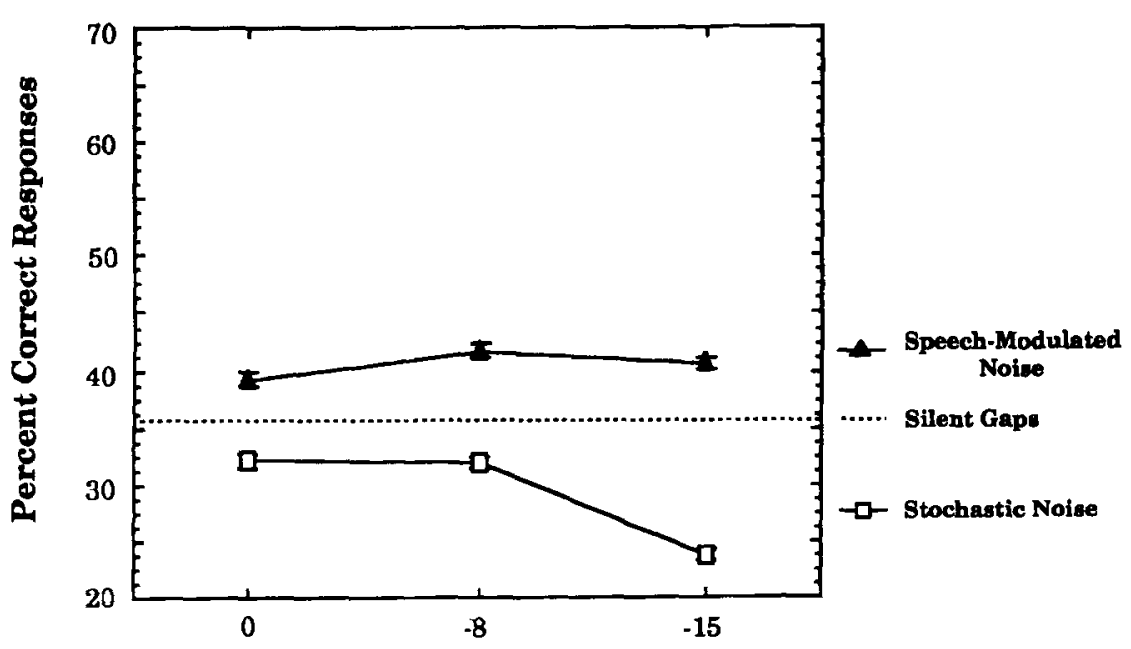

Signal-to-Noise Ratio (dB)

Figure 3. Mean percent repetition accuracy (and standard-error confidence intervals) for isolated monosyllables subjected to periodic interruption by silence, stochastic noise, or noise modulated by the amplitude envelope of obliterated speech fragments.

cant interrupter $\times$ block interaction $[F(54,1197)=0.54$, $p>$.99]. Subsequent Tukey tests indicated $(p<.05)$ that interpolation of stochastic noise reduced intelligibility relative to the silent-gap condition at all $\mathrm{S} / \mathrm{N}$ ratios, and that interpolation of speech-modulated noise increased intelligibility at $\mathrm{S} / \mathrm{N}$ ratios of -8 and $-15 \mathrm{~dB}$. Analysis of the block effect, using Helmhert single-df contrasts, indicated that repetition accuracy improved consistently across blocks of monosyllables ( $F \geq 5.94, p<.02$ or better) until reaching asymptote at the fifth block. This pattern of improvement, which resulted in an intelligibility increase of about $9 \%$ across blocks, held for all conditions, as indicated by the nonsignificant interrupter $\times$ block interaction.

The context provided by fragments of monosyllables adjacent to the interruptions was not sufficient to permit the restoration of intelligibility when interruptions were filled with stochastic noise-indeed, a reduction of intelligibility was observed when stochastic noise was added to the silent gaps. However, when the interrupting noise carried information concerning the broadband envelope of the deleted fragments, intelligibility was increased by an average of about $4 \%$ relative to the silent-gap condition. These opposing effects upon intelligibility observed with stochastic and speech-modulated noise illustrate an inherent tradeoff that always exists in conditions permitting phonemic restoration. Extraneous sound bursts that can provide sufficient input for synthesis of obliterated speech through auditory reallocation (Warren et al., 1994) are also effective backward and forward maskers of the adjacent intact speech fragments (e.g., see Bashford \& Warren, 1987a). Thus, the net intelligibility of an interruption condition will reflect a tradeoff between the extent of temporal masking of the intact speech and the extent to which the restoration mechanism can employ available bottom- up and top-down information for perceptual synthesis. The sentence fragments presented in Experiment 1 provided sufficient context to produce a net intelligibility increase for all six of the interpolated noise conditions examined, although the results do indicate that the $\mathrm{S} / \mathrm{N}$ ratio of $-8 \mathrm{~dB}$ produced an optimal tradeoff between restoration and temporal masking. In contrast, the isolated monosyllables provided insufficient context for restoration when interrupted by stochastic noise, resulting in a net loss of intelligibility at all S/N ratios. However, when stochastic noise was replaced by speech-modulated noise, so as to provide additional, bottom-up information concerning the amplitude envelopes of the deleted fragments of monosyllables, a cancellation of masking occurred, with intelligibility increasing by about $8 \%$ at $\mathrm{S} / \mathrm{N}$ ratios of 0 and $-8 \mathrm{~dB}$ and increasing by $17 \%$ at a $\mathrm{S} / \mathrm{N}$ ratio of $-15 \mathrm{~dB}$.

\section{GENERAL DISCUSSION}

Previous studies have revealed that the verbal form of auditory induction, known as phonemic restoration, is a highly adaptive and sophisticated process that can use intact portions of speech surrounding an interruption to identify and perceptually reconstruct the obliterated signal fragment. The results of the present study extend our understanding of the contextual factors governing this restorative process, and show that amplitude-envelope information available during an interruption is also used as a guide for perceptual synthesis. In Experiment 1, listeners were presented with periodically interrupted sentences, and it was found that filling gaps with stochastic noise produced an intelligibility increase ranging from $13 \%$ to $17 \%$, depending upon $\mathrm{S} / \mathrm{N}$ ratio, whereas interpolation of noise that followed the broadband amplitude envelope of the re- 
placed speech fragments produced an increase ranging from $28 \%$ to $38 \%$. Thus, amplitude information available during the interruptions in sentences contributed at least as much to phonemic restoration as did the intact portions of speech preceding and following the interruptions. In Experiment 2, listeners were presented with lists of interrupted monosyllabic words, and it was found that interpolation of stochastic noise produced only masking, with performance decreases ranging from $4 \%$ to $13 \%$, depending upon the $\mathrm{S} / \mathrm{N}$ ratio. However, when the broadband amplitude envelope of deleted fragments of monosyllables was superimposed upon the interpolated noise, there was a substantial cancellation of masking, yielding a net intelligibility increase of about $4 \%$ at all $\mathrm{S} / \mathrm{N}$ ratios. Thus, restoration of isolated monosyllables was possible, but only when context provided by intact speech fragments was augmented by information available during the interruptions.

In keeping with past studies, the restoration of intelligibility induced by interpolated stochastic noise in the present experiments depended upon the amount of contextual information provided by the remaining fragments of speech. Interpolation of stochastic noise in periodic gaps has been found not to enhance the intelligibility of monosyllables spoken in isolation (Bashford et al., 1992; Bashford \& Warren, 1987a; Dirks \& Bower, 1970; Miller \& Licklider, 1950), although monosyllables are subject to restoration when spoken in sentential context. For example, Bashford et al. (1992) presented listeners with periodically interrupted SPIN (speech perception in noise) sentences (Kalikow, Stevens, \& Elliott, 1977) and found that the intelligibility of the sentence-final, monosyllabic keywords was increased by about $10 \%$ in nonpredictive sentence contexts (e.g., "Ruth hopes she called about the junk") and by about $20 \%$ in predictive contexts (e.g., "Throw out all this useless junk") when periodic gaps in the sentences were filled with higher intensity 'stochastic noise $(\mathrm{S} / \mathrm{N}$ ratio $=-10 \mathrm{~dB})$ rather than silence. Thus, it appears that restoration of intelligibility requires linguistic information beyond that provided by the isolated syllable and is greatest when the speech consists of sentences providing not only intralexical coarticulation, intonation, and syntactic structure, but also semantic, top-down information. It should be noted that a similar contextual dependency has also been observed in studies examining the effects of interpolated noise upon the restoration of apparent continuity of speech. Thus, interpolation of higher amplitude stochastic noise in periodically spaced gaps may produce illusory continuity through very brief interruptions $(100-150 \mathrm{msec})$ when the verbal stimuli are isolated monosyllables (Bashford \& Warren, 1979, 1987b; Miller \& Licklider, 1950) or sentences read with the order of words reversed (Bashford \& Warren, 1987b), whereas normal prose permits restoration of apparent continuity through much longer noise-filled gaps of $300 \mathrm{msec}$ or more. Interestingly, the limiting gap duration closely corresponds to the average word duration in prose passages (Bashford et al., 1988; Bashford \& Warren, 1987b), with this correspondence being maintained when the playback rate of the same message is increased or decreased by $15 \%$ (Bashford et al., 1988).

As discussed briefly in the introduction, Warren and his co-workers (Warren, 1982, 1984; Warren et al., 1994) have attributed the restoration of obliterated speech to the linguistic form of a general auditory process that restores continuity of interrupted signals by using a portion of the neural input from the interrupting noise to reconstruct contextually appropriate missing fragments. Since this process of auditory reallocation requires that the spectral representation of the interrupting sound overlap that of the speech, restoration is limited to verbal components that could have been masked by the noise. This theory is supported by previous studies showing that when narrowband speech $(1.5-\mathrm{kHz}$ center frequency) is interrupted by narrowband stochastic noise with various center frequencies, the extent of restoration, whether measured by intelligibility (Bashford et al., 1992; Bashford \& Warren, 1979) or by the upper durational limit of apparent continuity (Bashford \& Warren, 1987b), depends upon the masking potential of the particular noise band. Moreover, direct evidence for reallocation under these conditions was subsequently provided by Warren et al. (1994), who found that there was a reciprocal interaction of narrowband speech and interpolated narrowband stochastic noise, such that a loudness reduction of the noise occurred that was commensurate with its ability to induce illusory continuity of the speech (cf. Bashford \& Warren, 1987b). Experiment 1 of the present study, in which sentence intelligibility was highest with interrupting noise at $-8 \mathrm{~dB} \mathrm{~S} / \mathrm{N}$, has provided further evidence consistent with the reallocation theory and with the results of previous studies. Verschuure (1978), for example, examined conditions permitting optimal continuity of discourse with an on-time of periodic interruption $(125 \mathrm{msec})$ similar to that used in Experiment $1(150 \mathrm{msec})$ and found that a $\mathrm{S} / \mathrm{N}$ ratio of $-10 \mathrm{~dB}$ provided an optimal balance between masking and the restoration of continuity. Somewhat similar results were obtained by Powers and Wilcox (1977), who examined effects of noise level upon the intelligibility of periodically interrupted CID sentences and found that intelligibility was highest at $\mathrm{S} / \mathrm{N}$ ratios of -6 to $-24 \mathrm{~dB}$. This range of $\mathrm{S} / \mathrm{N}$ ratios producing equivalent intelligibility includes relative noise levels higher than that found to be optimal in the present study, but it is likely that this difference was due to the much longer on-time ( $333 \mathrm{msec})$ used by Powers and Wilcox, which would be expected to permit proportionately less forward and backward temporal masking of speech fragments adjacent to the noise and, hence, to result in a broader peak in the $\mathrm{S} / \mathrm{N}$ function.

Finally, the effects of amplitude envelope in the present study were both substantial and immediate, requiring no practice or learning by the listener. This suggests that normal speech processing involves not only a narrowband analysis of spectral information but also a wideband integration or summing of amplitude levels across spectral regions. This conclusion is consistent with hypotheses advanced by several investigators (e.g., Erber, 1979; Rosen, 
1992; van Tasell et al., 1987) and is also consistent with previous findings indicating that amplitude-envelope cues, superimposed upon noise or tones, can supplement speech identification when combined with other sources of information (for a review, see Repp et al., 1992). The impact of envelope cues in the present study indicates that use of this information by listeners is routine, and that the use of speech-modulated noise for such purposes as phonemic restoration studies (e.g., Samuel, 1987, 1991; Samuel \& Ressler, 1986) may introduce bottom-up information concerning the identity of missing speech fragments.

\section{REFERENCES}

American National Standards Institute (1971). USA standard method for measurement of monosyllabic word intelligibility [ANSIS3.2, 1960 (R1971)]. New York: Author.

Bashford, J. A., JR., Meyers, M. D., Brubaker, B. S., \& Warren, R. M. (1988). Illusory continuity of interrupted speech: Speech rate determines durational limits. Journal of the Acoustical Society of America, 84, 1635-1638.

BAShFord, J. A., JR., Riener, K. R., \& WARREN, R. M. (1992). Increasing the intelligibility of speech through multiple phonemic restorations. Perception \& Psychophysics, 51, 211-217.

Bashford, J. A., JR., \& Warren, R. M. (1979). Perceptual synthesis of deleted phonemes. In J. J. Wolf \& D. H. Klatt (Eds.), Speech communication papers (pp. 423-426). New York: Acoustical Society of America.

BASHFORD, J. A., JR., \& WARREN, R. M. (1987a). Effects of spectral alternation on the intelligibility of words and sentences. Perception \& Psychophysics, 42, 431-438.

BASHFord, J. A., JR., \& WARren, R. M. (1987b). Multiple phonemic restorations follow the rules for auditory induction. Perception \& Psychophysics, 42, 114-121

BECK, W. G., \& SPEAKS, C. S. (1993). Intelligibility of selected passages from the speech intelligibility rating (SIR) test. Journal of Speech \& Hearing Research, 36, 1075-1082.

Blamey, P. J., Martin, L. F. A., \& Clark, G. M. (1985). A comparison of three speech coding strategies using an acoustic model of a cochlear implant. Journal of the Acoustical Society of America, 77, 209-217.

BREEUWER, M., \& PlOMP, R. (1984). Speechreading supplemented with frequency-selective sound-pressure information. Journal of the Acoustical Society of America, 76, 686-691.

BREEUWER, M., \& PloMP, R. (1986). Speechreading supplemented with auditorily presented speech parameters. Journal of the Acoustical Society of America, 79, 481-499.

Cherry, E., \& WILEY, R. (1967). Speech communications in very noisy environments. Nature, 214, 1164

Ciocca, V., \& Bregman, A. S. (1987). Perceived continuity of gliding and steady-state tones through interrupting noise. Perception \& Psychophysics, 42, 476-484.

Dannenbring, G. L. (1976). Perceived auditory continuity with alternately rising and falling frequency transitions. Canadian Journal of Psychology, 30, 99-114.

DeWitT, L. A., \& Samuel, A. G. (1990). The role of knowledge-based expectations in music perception: Evidence from musical restoration. Journal of Experimental Psychology: General, 119, 123-144.

DiRKS, D. D., \& BowER, D. (1970). Effect of forward and backward masking on speech intelligibility. Journal of the Acoustical Society of America, 47, 1003-1007.

Erber, N. P. (1972). Speech-envelope cues as an acoustic aid to lipreading for profoundly deaf children. Journal of the Acoustical Society of America, 51, 1224-1227.

ERBER, N. P. (1979). Speech perception by profoundly hearing-impaired children. Journal of Speech \& Hearing Disorders, 44, 255-270.

FESTEN, J. M., \& PLOMP, R. (1990). Effects of fluctuating noise and interfering speech on the speech reception threshold for impaired and normal hearing. Journal of the Acoustical Society of America, 88, $1725-1.736$
Frost, R. (1991). Phonetic recoding of print and its effect on the detection of concurrent speech in amplitude-modulated noise. Cognition, 39, 195-214.

Frost, R., RePP, B. H., \& Katz, L. (1988). Can speech perception be influenced by simultaneous presentation of print? Journal of Memory \& Language, 27, 741-755.

GORDON, P. C. (1988). Induction of rate-dependent processing by coarsegrained aspects of speech. Perception \& Psychophysics, 43, 137-146.

Grant, K. W., Ardell, L. H., Kuhl, P. K., \& Sparks, D. W. (1985). The contribution of fundamental frequency, amplitude envelope, and voicing duration cues to speechreading in normal-hearing subjects. Journal of the Acoustical Society of America, 77, 671-677.

Grant, K. W., Braida, L. D., \& RenN, R. J. (1991). Single band amplitude envelope cues as an aid to speechreading. Quarterly Journal of Experimental Psychology, 43A, 621-645.

Grant, K. W., Braida, L. D., \& Renn, R. J. (1994). Auditory supplements to speechreading: Combining amplitude envelope cues from different spectral regions of speech. Journal of the Acoustical Society of America, 95, 1065-1073.

HaYs, W. L. (1988). Statistics. Chicago: Holt, Rinehart \& Winston.

Holloway, C. M. (1970). Passing the strongly voiced components of noisy speech. Nature, 226, 178-179.

HoRII, Y., HOUSE, A. S., \& HUGHES, G. W. (1971). A masking noise with speech-envelope characteristics for studying intelligibility. Journal of the Acoustical Society of America, 49, 1849-1856

Houtgast, T. (1972). Psychophysical evidence for lateral inhibition in hearing. Journal of the Acoustical Society of America, 51, 1885-1894.

Kalikow, D. N., Stevens, K. N., \& Elliott, L. L. (1977). Development of a test of speech intelligibility in noise using sentence materials with controlled word predictability. Journal of the Acoustical Society of America, 61, 1337-1351.

KLUENDER, K. R., \& JENISON, R. L. (1992). Effects of glide slope, noise intensity, and noise duration on the extrapolation of FM glides through noise. Perception \& Psychophysics, 51, 231-238.

LAYTON, B. (1975). Differential effects of two nonspeech sounds on phonemic restoration. Bulletin of the Psychonomic Society, 6, 487-490.

LemaY, D. G., \& BraidA, L. D. (1994). Band-envelope speechreading supplements derived from noisy speech [Abstract]. Journal of the Acoustical Society of America, 95, 3014.

Miller, G. A., \& Licklider, J. C. R. (1950). The intelligibility of interrupted speech. Journal of the Acoustical Society of America, 22, 167-173.

PowERs, G. L., \& WILcox, J. C. (1977). Intelligibility of temporally interrupted speech with and without intervening noise. Journal of the Acoustical Society of America, 61, 195-199.

REMEZ, R. E., \& RUBin, P. E. (1990). On the perception of speech from time-varying acoustic information: Contributions of amplitude variation. Perception \& Psychophysics, 48, 313-325.

REPP, B. H. (1992). Perceptual restoration of a "missing" speech sound: Auditory induction or illusion? Perception \& Psychophysics, 51, 14-32.

RePP, B. H., Frost, R., \& Zsiga, E. (1992). Lexical mediation between sight and sound in speechreading. Quarterly Journal of Experimental Psychology, 45A, 1-20

ROSEN, S. (1992). Temporal information in speech: Acoustic, auditory and linguistic aspects. Philosophical Transactions of the Royal Society of London, 336, 367-373.

Salasoo, A., \& Pisoni, D. B. (1985). Interaction of knowledge sources in spoken word identification. Journal of Memory \& Language, 24, 210-231.

Samuel, A. G. (1981a). Phonemic restoration: Insights from a new methodology. Journal of Experimental Psychology: General, 110, 474-494.

Samuel, A. G. (1981b). The role of bottom-up confirmation in the phonemic restoration illusion. Journal of Experimental Psychology: Human Perception \& Performance, 7, 1124-1131.

Samuel, A. G. (1987). Lexical uniqueness effects on phonemic restoration. Journal of Memory \& Language, 26, 36-56.

SAMUEL, A. G. (1991). A further examination of attentional effects in the phonemic restoration illusion. Quarterly Journal of Experimental Psychology, 43A, 679-699.

Samuel, A. G., \& Ressler, W. H. (1986). Attention within auditory word perception: Insights from the phonemic restoration illusion. Jour- 
nal of Experimental Psychology: Human Perception \& Performance, 12, 70-79.

SASAKI, T. (1980). Sound restoration and temporal localization of noise in speech and music sounds. Tohuku Psychologica Folia, 39, 79-88.

SCHROEDER, M. R. (1968). Reference signal for signal quality studies. Journal of the Acoustical Society of America, 44, 1735-1736.

Silverman, S. R., \& Hirsh, I. J. (1955). Problems related to the use of speech in clinical audiometry. Annals of Otology, Rhinology, \& Laryngology, 64, 1234-1245.

THURLOW, W. R. (1957). An auditory figure-ground effect. American Journal of Psychology, 70, 653-654.

Trout, J. D., \& Poser, W. J. (1990). Auditory and visual influences on phonemic restoration. Language \& Speech, 33, 121-135.

van Tasell, D. J., Soli, S. D., Kirby, V. M., \& Widin, G. P. (1987). Speech waveform envelope cues for consonant recognition. Journal of the Acoustical Society of America, 82, 1152-1161.

VERSCHUURE, J. (1978). Auditory excitation patterns: The significance of the pulsation threshold method for the measurement of auditory nonlinearity. Unpublished doctoral dissertation, Erasmus University, Rotterdam.

VERSCHUURE, J., \& BROCAAR, M. P. (1983). Intelligibility of interrupted meaningful and nonsense speech with and without intervening noise. Perception \& Psychophysics, 33, 232-240.

WALLEY, A. C. (1988). Spoken word recognition by young children and adults. Cognitive Development, 3, 137-165.

WARREN, R. M. (1970). Perceptual restoration of missing speech sounds. Science, 167, 392-393.

WARREN, R. M. (1982). Auditory perception: A new synthesis. New York: Pergamon.

WARREN, R. M. (1984). Perceptual restoration of obliterated sounds. Psychological Bulletin, 96, 371-383.

Warren, R. M., Bashford, J. A., JR., Healy, E. W., \& Brubaker, B. S.
(1994). Auditory induction: Reciprocal changes in alternating sounds. Perception \& Psychophysics, 55, 313-322.

WARren, R. M., \& OBUSEK, C. J. (1971). Speech perception and phonemic restorations. Perception \& Psychophysics, 9, 358-362.

WARREN, R. M., Obusek, C., \& ACKroff, J. M. (1972). Auditory induction: Perceptual synthesis of absent sounds. Science, 176, 1149-1151.

Warren, R. M., \& Sherman, G. L. (1974). Phonemic restorations based on subsequent context. Perception \& Psychophysics, 16, 150-156.

\section{NOTES}

1. In keeping with the observations of Frost and his colleagues (Frost, 1991; Frost et al., 1988), our laboratory personnel, who were highly familiar with the CID sentences employed in Experiment 1, found that they could easily identify the sentence used to modulate the amplitude of a given noise segment. To assess intelligibility in the absence of prior knowledge of the stimuli, we presented the modulated noises to 2 experienced listeners who had never heard the original sentences. One listener declined even to guess for any of the stimuli; the other listener managed to identify correctly only 2 of the 500 keywords within the sentences. This yielded an estimated average intelligibility of just $0.2 \%$ for the 2 listeners.

2. Previous studies of phonemic restoration under conditions of periodic interruption have employed a 50\% duty cycle of switching, and this duty cycle was employed for interrupting word lists in Experiment 2. However, we departed from this procedure in Experiment 1 and used a reduced percentage of on-time $(37.5 \%)$ for the CID sentences to avoid possible ceiling effects-we have previously found intelligibility scores for these sentences to be about $85 \%$ when interrupted at $200 \mathrm{msec}$ on/off by interpolated stochastic noise (Bashford et al., 1992).

(Manuscript received August 29, 1994; revision accepted for publication July 31,1995 .) 